\title{
Hybrid Extendable Linear Actuators: Design and Applications
}

\author{
Mohamed Tahir Shoani ${ }^{1}$, Mohamed Najib Ribuan ${ }^{1 *}$ and Ahmad Athif Faudzi ${ }^{2}$ \\ ${ }^{1}$ Faculty of Electrical and Electronic Eng., Universiti Tun Hussein Onn Malaysia, Parit Raja, Johor Bahru, 86400, Malaysia. \\ ${ }^{2}$ School of Electrical Engineering, Universiti Teknologi Malaysia, 81310 UTM Skudai, Johor, Malaysia.
}

*Corresponding author: mnajib@uthm.edu.my, Tel: +607-4538333

\begin{abstract}
There are many types of different actuators in the field of robotics. For applications where a linear displacement is required, a linear extendable actuator would be the method of choice. The hybrid class of extendable actuators possesses unique features making it suitable for many applications where the soft and rigid types fall short. However, there has not been a clear designation and classification of extendable linear hybrid actuators in the literature. This paper addresses this matter and provides the first overview of the hybrid class of extendable actuators. The paper performs a categorization and characterization of this class of extendable linear actuators based on their method of operation as well as the inherent unique features separating them from the rest. The paper contains five sections, and three sub-sections pertaining to the different categories of Hybrid actuators, and their applications. New research in this field continues to add features to this class of actuators through improvements and added capabilities.
\end{abstract}

Keywords: Hybrid, Extendable, Linear, Stroke, Actuator.

(C) 2019 Penerbit UTM Press. All rights reserved

Article History: received 4 September 2019; accepted 1 December 2019; published 24 December 2019.

\section{INTRODUCTION}

In the field of robotics, actuators are used to affect the environment or perform an action required by the robot to achieve the prescribed or required outcome. Although many types of actuators are used depending on the environment and type of the robot, they can be broadly categorized as displacement and non-displacement actuators. Displacement actuators provide motion such as motors, hydraulic and pneumatic cylinders [1], while non-displacement actuators provide other effects such as light, sound, or other effects [2].

Displacement type actuators can be subcategorized into extendable and non-extendable actuators. An extendable actuator provides translational motion, while non-extendable actuators may provide rotary or other forms of motions such as bending, flowing or multidirectional [3].

Extendable linear actuators can be further categorized based on their body's flexibility to soft, rigid and hybrid. Soft actuators such as the McKibben Actuator [4][5], the Bellows Actuator [6], Ionic Electro-Active Polymers [4][7], Field-Activated Electro-Active Polymers [7][8] and others have a flexible body. Rigid actuators on the other hand have a stiff body, usually made from metal or other hard material. Examples of rigid actuators are electrical linear actuators, pneumatic and hydraulic cylinders [9][10] as well as piezoelectric [11] and magnetostrictive [12] actuators. Hybrid extendable linear actuators use flexible material that forms a rigid column once extended. This is in contrast to linear soft actuators that remain relatively soft after actuation, unless hardened by jamming [13][14] or other techniques. Linear extendable hybrid actuators present certain advantages over their soft and hard counterparts.

The next section explores the operation and characteristics of hybrid extendable linear actuators as follows. Sub-section one will focus on the direct roll-out extension type. Sub-section two will focus on the rotaryspiral extension type. Sub-section three will focus on hybrid actuators that extend through growth. Sub-section four will focus on unfolding hybrid actuators, and finally, sub-section five will focus on hybrid actuators that extend by merging pieces together.

\section{TYPES OF HYBRID ACTUATORS}

There are five types of Hybrid Linear actuators, each of which applies a different approach to protrude an extended column. These types also differ in their characteristics; each with its own strengths and weaknesses. In the following sections, the five different types shall be surveyed.

\subsection{Direct Roll-Out Extension}

Hybrid roll-out actuators that extend the stored flexible material at a 90-degree angle to the base are referred to as Direct Roll-Out Extension (DROX) actuators. In this type of actuators, the flexible part that would be used to form the column is stored as a roll inside the main actuator body. After being rolled out of the housing, the soft material changes its cross section to gain more bending strength when extended. Depending on the cross section 
(subtended angle), a DROX actuator can be a classified as a Storable Tubular Extendable Member (STEM), a slittube or a tape-spring [15].

DROX actuators have the advantage of a large extension ratio, however, depending on the design; they exhibit a varying weakness towards bending stress when extended. More recent research has focused on solving other problems related to DROX actuators such as eliminating blossoming and improving repeatability and precision by tensioning the coiled boom [16].

\subsubsection{Storable Tubular Extendable Member (STEM)}

STEM actuators are composed of one or more tubes that overlap, hence the subtended angle (radius of curvature) ' $a$ ' is greater than 360 , giving it a higher stiffness with respect to slit-tube or tape spring designs.

Extendable actuators utilizing the STEM design have been introduced in many variations; the main difference being the number of layers used and/or the cross section of these layers after extrusion from the base of the actuator. In [17] many variations of the STEM design are shown, utilizing single, double, or a triple-reel system.

STEM actuators have been used in space for many applications, such as antenna deployment, docking booms, grasping devices and others [18][17][15][19][20]. STEM actuators have also been used in other applications. In [21], two STEMs are used to form a two D.O.F. parallel robot, while [22] presents a space telescope in which the secondary mirror is stowed during launch and deployed when in orbit using six extendable actuators (feet), which are also used to correct positional errors of the secondary mirror.

An interesting realization of the STEM approach is the zipper-mast [23]. This actuator joins three individually stored (rolled-up) strips of spring-steel to form a rigid column when extended. The strips are progressively notched such that they interlock with each other upon roll-out, forming a rigid column with a triangular crosssection. The advantage of this approach is the relative rigidity, allowing it to support loads such as cameras or actuators at its end. The weakness of this actuator, however, is that lateral forces causing a large-enough moment may disengage the interlock between the sections resulting in a mechanical failure. Although this can be avoided by limiting the height, or remedied by rewinding, and then rolling out the sections to reform the connections, it limits the application of this actuator to low load, low height (low extendibility), or low-speed (low mobility) functions.

\subsubsection{Slit-Tube}

A slit-tube design is similar to a STEM; however, the cross section does not form a complete circle, and hence the subtended angle ' $a$ ' is less than 360 , but greater than 225 degrees:

$$
225^{\circ} \leq \alpha \leq 360^{\circ}
$$

Compared to STEM, slit-tube actuators are less rigid; they withstand less bending and torsional forces. In [24] the power of the stored strain energy in the composite slit-tube is used to deploy a folded solar array in space.
Compared to standard rigid solar panel arrays, the $5.4 \mathrm{x}$ 1.7 meter solar array is $33 \%$ lighter and has a volume reduction factor of 4 .

\subsubsection{Tape-Spring}

Tape spring designs are similar to a measuring-tape (aka tape-measure). In this type of actuators, the subtended angle ' $a$ ' is less than 225 degrees; therefore, tape springs have the lowest bending and torsional strength among DROX Hybrid actuators. For this reason, they are mainly used for applications which do not require high lateral rigidity.

In [25] a retractable arm, fabricated from an isotropic material, is used to collect samples from soil and other material. The motion of the arm is vertical, avoiding lateral forces which may result in undesired bending or collapse of the actuator column.

\subsection{Rotary-Spiral Extension}

Rotary-spiral extension (RSX) actuators are similar to DROX actuators; however, they differ in the way the extended part is protruded outwards. While DROX actuators extend directly from the base, RSX actuators protrudes the extending section in a spiral manner and employ specific mechanisms to join the protruding strip to form a linear column.

There are several realizations of RSX actuators. In [26] a long-stroke linear actuator based on a previous patent [27]; (manufactured by 'Paco Spiralift'), forms a rigid column by interlocking a wind-up band and a toothspring in a helical manner. The perforated band and the toothed helical spring are both stored at the base. A mechanical arrangement interlocks the two as they extrude from the base forming a solid column. The advantages of this actuator are its rigid structure and heavy load bearing, resulting in excellent performance when it comes to applications such as stage lifting or other heavy loads, as well as a high bending strength. However, it is relatively heavy, making it unattractive for space or mobile applications.

The spiral-zipper [28] is another RSX linear actuator that converts a plastic band into a spiral-column through meshing the top and bottom teeth (cut perforations) with the next level. The advantages of this approach are its light weight and compression bearing ability, however it has weak bending and tension resistance as well as being slow and less rigid while extended, making it unstable (wobbly). Such an actuator would be useful for space applications where light weight is an advantage and the absence of gravity reduces the problem of pliability.

\subsection{Rotary Growth Extension}

This category of hybrid actuators employs a special technique in which material is extruded by a rotating nozzle to deposit a thermosetting material around and behind the actuator's tip, creating layers that push the tip deeper into the soil or other medium. One of the main differences between this approach and the rest of hybrid actuators is that this actuator is non-retractable. Once the actuator has extruded, it's almost impossible to retract it; analogous to tree roots. 
In [29] and [30], a growing device employing this approach is introduced. The device uses a polypropylene filament to (3D) print its body as a root in the form of a tube while penetrating its environment. The device employs a rotary motion, but changes direction every few cycles to prevent the twisting of the filament and power wires that are connected to the trunk.

\subsection{Extension through Unfolding}

Another category of hybrid linear actuators employs an origami approach to extend and retract the actuator column. The flexible material allows the actuator to bend and retract or extend, while the origami technique of perpendicular folding allows it to become rigid once extended. This Extension through Unfolding (XTU) category of actuators is compact, lightweight, and scalable.

The foldable robotic arm [31] utilizes this approach to enable a UAV to perform tasks such as picking items from tight locations, between tree branches or from underwater.

\subsection{Extension through Piecing}

The final category of hybrid linear actuators is interesting. It joins similar pieces of rigid material to form the extended rigid column and is referred to as an Extension through Piecing (XTP) actuator. This class of actuators is different in that the pieces are also rigid while in storage, however, they are connected to a chain which makes the stored structure flexible.

The Kataka ('Golden Chain' in Sanskrit) actuator [32] employs this technique. Its extended part is an elongated spindle member made up by interlocking many parts joined up as a chain link. The inventor claims it has an extension ratio of up to $1: 20$, and can withstand high loads, bending stress as well as shear stress. Although this has been mentioned on the internet [33][34], no literature was found reporting an experimental validation.

\section{CONCLUSION}

In this paper, a review of hybrid linear actuators was conducted. Five types were investigated, with the first one containing three sub-types. The distinction between the different kinds is based on the working principle, while the subcategorization of the first section was based on the minor differences in the design.

The Direct Roll-Out Extendable (DROX) actuators are simpler and more widespread that the other types, while the Rotary Spiral Extension (RSX) type is used for precision lifting of heavy loads. The Rotary Growth Extension (RGX) can create solid roots in the ground and can be useful for tunneling or soil exploration. The XTU type of hybrid actuators is characterized by its simplicity and lightweight which allows it to be carried by a UAV for various tasks. Finally, the XTP actuator's high-power density and extended reach makes it a good candidate for replacing pneumatic and hydraulic cylinders when extra elongation is required.

When compared to rigid linear actuators, the hybrid class demonstrates a higher extension ratio than electrical, hydraulic and pneumatic types. Hybrid actuators also outperform soft actuators in terms of precision and controllability.

\section{ACKNOWLEDGMENT}

This research is supported by the following grants:

- Research Management Centre (RMC) Universiti Tun Hussein Onn Malaysia (UTHM) under the Short Term Grant Scheme (Code U640)

- Ministry of Higher Education - Research University Grant: Q.J130000.3023.01M04.

\section{REFERENCES}

[1] J. M. Hollerbach, "A Comparative Analysis of Actuator technologies for Robotics," Robot. Rev. 2, 1990.

[2] D. Cook, Robot Building for Beginners. Springer, 2015.

[3] B. L. Wang and F. Iida, "Deformation in Soft-Matter Robotics," no. September, pp. 125-139, 2015.

[4] J. T. Godfrey, "Soft Robotic Actuators," 2017.

[5] A. A. M. Faudzi, J. Ooga, T. Goto, M. Takeichi, and K. Suzumori, "Index Finger of a Human-like Robotic Hand using Thin Soft Muscles," IEEE Robot. Autom. Lett., vol. 3, no. 1, pp. 1-1, 2018.

[6] G. Immega, "Bellows actuator," 1993.

[7] K. J. Kim and S. Tadokoro, Electroactive Polymers for Robotic Applications. 2007.

[8] F. B. Madsen, A. E. Daugaard, S. Hvilsted, and A. L. Skov, "The Current State of Silicone-Based Dielectric Elastomer Transducers," Macromol. Rapid Commun., vol. 37, no. 5, pp. 378-413, 2016.

[9] J. E. Huber, N. A. Fleck, and M. F. Ashby, "The selection of mechanical actuators based on performance indices," Proc. R. Soc. A Math. Phys. Eng. Sci., vol. 453, no. 1965, pp. 2185-2205, 1997.

[10] G. Granosik and J. Borenstein, "Pneumatic actuators for serpentine robots," Proc. 8th Int. Conf. Walk. Climbing Robot. (\{CLAWAR 2005\}), pp. 719-726, 2005.

[11]C. Niezrecki, D. Brei, S. Balakrishnan, and A. Moskalik, "Piezoelectric actuation: State of the art," Shock Vib. Dig., vol. 33, no. 4, pp. 269-280, 2001.

[12]H. Search et al., "Modeling and optimization of magnetostrictive actuator amplified by compliant mechanism," 2017.

[13]N. G. Cheng et al., "Manipulator Enabled by Jamming of Granular Media," Proc. 2012 IEEE Int. Conf. Robot. Autom., pp. 4328-4333, 2012.

[14]A. Jiang and T. Aste, "Granular jamming with hydraulic control," Asme Idetc, no. Idetc, pp. 1-7, 2013.

[15] A. Lee and J. M. Fernandez, "Mechanics of Bistable Two-Shelled Composite Booms," 2018 AIAA Spacecr. Struct. Conf., no. January, pp. 1-24, 2018.

[16] A. J. Daton-Lovett, "Extendible member," 2001.

[17]P. L. Conley, Space Vehicle Mechanisms: Elements of Successful Design. John Wiley \& Sons, 1998.

[18]F. Rehnmark, M. Pryor, B. Holmes, D. Schaechter, N. Pedreiro, and C. Carrington, "Development of a Deployable Nonmetallic Boom for Reconfigurable Systems of Small Spacecraft," Proc. Struct. Struct. Dyn. Mater. Conf., pp. 1-19, 2007. 
[19]B. K. H. Iguchi et al., "Achievement of Long-term On-orbit Operation of the SIMPLE Inflatable Extension Mast," vol. 14, pp. 13-17, 2016.

[20] K. Higuchi, K. Watanabe, A. Watanabe, H. Tsunoda, and H. Yamakawa, "Design and evaluation of an ultra-light extendible mast as an inflatable structure," AIAA/ASME/ASCE /AHS/ASC Struct. Struct. Dyn. Mater. Conf., no. AIAA-2006-1809, pp. 1-7, 2006.

[21]S. Seriani and P. Gallina, "A Storable Tubular Extendible Member (STEM) parallel robot: Modelization and evaluation," Mech. Mach. Theory, vol. 90, pp. 95-107, 2015.

[22]L. Blanchard, "A Tape-Spring Hexapod for Deployable Telescopes: Dynamics," ESA Spec. Publ., vol. 2006, no. June, pp. 27-30, 2006.

[23] G. Woodruff, P. Muench, and G. Witus, "Zipper mast for enhanced communications and surveillance," Proc. SPIE, vol. 8045, no. May 2011, p. 804512, 2018.

[24] J. Banik, S. Kiefer, M. Lapointe, and P. Lacorte, "On-orbit validation of the roll-out solar array," IEEE Aerosp. Conf. Proc., vol. 2018-March, pp. 19, 2018.

[25] W. Lu, A. Song, and F. Hao, "Design and analysis of spring roll-style retractable sampling arm," 2010 IEEE Int. Conf. Inf. Autom. ICIA 2010, pp. 23742378, 2010.
[26] S. won Lee, O. Kwon, and K. H. Rim, "Analysis and design of long-stroke linear actuators," J. Mech. Sci. Technol., vol. 28, no. 8, pp. 3197-3202, 2014.

[27] A. Agent and F. D.- Gil, "Push actuator," 1989.

[28]F. Collins and M. Yim, "Design of a spherical robot arm with the Spiral Zipper prismatic joint," Proc. IEEE Int. Conf. Robot. Autom., vol. 2016-June, pp. 2137-2143, 2016.

[29]A. Sadeghi, A. Tonazzini, L. Popova, and B. Mazzolai, "A novel growing device inspired by plant root soil penetration behaviors," PLoS One, vol. 9, no. 2, pp. 1-10, 2014.

[30] A. Sadeghi, A. Mondini, and B. Mazzolai, "Toward Self-Growing Soft Robots Inspired by Plant Roots and Based on Additive Manufacturing Technologies," Soft Robot., vol. 00, no. 00, p. soro.2016.0080, 2017.

[31] S.-J. Kim, D.-Y. Lee, G.-P. Jung, and K.-J. Cho, “An origami-inspired, self-locking robotic arm that can be folded flat," Sci. Robot., vol. 3, no. 16, p. eaar2915, 2018.

[32] J. J. Sorensen, "Method and apparatus for making an elongate spindle member of chain links for transfer of pressure and tensile loads," 2006.

[33] K. J. Jorgensen, "KATAKA Profile.".

[34] Kataka1, "The principle behind KATAKA linear actuators." 